\title{
On an Architectural Concept for Didactics in the Context of Constructive Alignment
}

\author{
Axel Böttcher, Veronika Thurner \\ Department of Computer Science and Mathematics, Munich University of Applied Sciences, \\ Germany.
}

\begin{abstract}
In the wake of the massive digitalization in education brought about by the Covid-19 pandemic, a vast variety of didactic concepts, methods, activities, tools and technologies came into use and are now widely discussed. However, a lack of clarity is observable, as to how these different concepts, activities and tools integrate into a big picture, when to use what, and which aspects have to be considered and addressed to create solutions that work effectively towards achieving specific learning and teaching objectives. To systematically structure this vast variety of didactic concepts, activities and tools, we introduce an architectural concept for didactics in the context of constructive alignment. We achieve this via an abstraction process, which clusters the existing didactic concepts, activities and tools, and specifies interrelations between the identified clusters. On this basis, it is possible to quickly adapt our model by subtractive customization, thus focusing on what is really needed within a specific teaching and learning situation.
\end{abstract}

Keywords: Teaching methods; didactic design; learning technologies. 


\section{Introduction and Motivation}

The Covid-19-pandemic and correlated lockdowns induced teachers and lecturers worldwide to transfer their teaching and learning activities from on site or hybrid into purely virtual formats. Due to the highly acute crisis, this transition had to be realized extremely quickly, to ensure maximum health safety while minimizing educational losses in our learners. Even though the transition to virtual teaching went remarkably well in many instances, it nevertheless shows traits of "emergency remote teaching", thus leaving room for systematic improvement that should be invested before these emergency induced approaches establish themselves as general practice.

In many cases, additional funding was provided to support these efforts, which expedited the increasing divergence of didactic concepts, methods and tools. However, these new ways and means also create several new challenges for all stakeholders involved in providing higher education:

- As lecturers, the general setting for executing our everyday work is expanded by an additional dimension, which creates a host of new options for doing our job. Facing these new options entails the challenge of unerringly selecting from this multitude a subset that is suitable to promote one's teaching and learning objectives within a specific setting, and then getting this subset into practice.

- For decision makers in educational strategy and politics, an everlasting question is where to invest the usually scant monetary resources, in order to achieve the best possible benefit. A well observed pattern is to spend large sums of money on buying technological devices and then medially brag about it to generate publicity. (Note that as technology tends to be haptic, it is more tangible and thus easier to brag about than e.g. didactic methods, which tend to be purely abstract.) However, if these investments are not founded on and aligned with appropriate goals, conceptual methods and the enablement of all parties involved, the new equipment will not bring about the intended benefit.

- The organizational dimension of a university needs to take into account that the professors' main area of expertise usually lies within their technical domains, rather than e.g. in media design, tool usage or the development of didactic methods. Thus for all of these areas, lecturers need enablement and support which the institution ideally should provide, well-adjusted to the lecturers' actual needs.

To tackle these challenges, we applied established modeling methods from software engineering to structure the landscape of didactic concepts and topics into different dimensions, and define interrelations between these different aspects. (So we applied the 
engineering approach of the domain of our technical expertise to design our teaching and learning activities as well.)

The result of these efforts is an architectural concept for modern didactics, which can be easily customized to ones' individual needs. Thus, this work helps all the above stakeholders by creating some sort of map that provides orientation in the vast domain of didactic concepts, teaching and learning methods, media and tooling.

\section{Related Work}

Starting point is the concept of Constructive Alignment, whose principles are described by Biggs and Tang as being the foundation for modern constructivist didactics (Biggs \& Tang, 2011). According to that model, intended learning outcomes (ILOs), teaching and learning activities (TLA), as well as assessment must be carefully aligned (cf. Figure 1). They describe the necessity of a systematic transition from ILOs to methods for TLAs. However, they do not mention the possibility of considering didactic intentions (such as motivating or activating students) that might be involved when selecting a sequence of methods during the design of teaching and learning activities (e. g. lectures, or units for self-regulated learning).

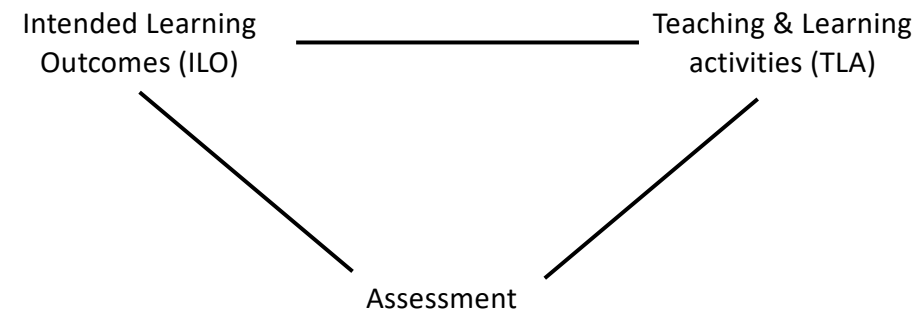

Figure 1. Constructive Alignment of Intended Learning Outcomes, Teaching/Learning Activities, and Assessment.

The question of how to systematically deduce appropriate TLAs from previously defined ILOs seems to be under-researched, even though it is an essential aspect of our everyday teaching efforts. Therefore, we focus on that path from ILOs to TLAs in this paper. Note that similarly, the systematic deduction of assessments that constructively align with ILOs and TLAs is non-trivial as well and should be subject of further research.

The SAMR-model (Puentedura, 2006) adds the aspect of technology to support TLAs, thus enabling a variety of TLAs that are not only enhanced by technology, but only become feasible because of technology. However, the SAMR-model raises several challenges, one of them being the risk to focus on generated products rather than on the students' learning process behind this product generation (Hamilton, Rosenberg, \& Akcaoglu, 2016). 
As well, several sites (Lathan, n.d.), (e-teaching.org) list more or less comprehensive selections of didactic concepts or teaching methods, but tend to intermingle TLAs, media or even the artefact generation process.

The Global Learning Landscape (Brothers \& Spies, 2020) aims at providing a taxonomy for innovation that happens in education. As such, the work is a high-level view and does not focus on details. However, the interactive design of the web-site is inspiring with respect to its visualization of the relationships between detailed didactic issues.

\section{Architectural Concept}

The core question in teaching design boils down to "What do I address how, and why?". The "why" denotes the didactic function or goal, whereas the "how" corresponds to both the method and the media and tools involved. Embedding these aspects and their interrelation into the principle of Constructive Alignment leads to our model (see Figure 2 for an overview), which aims at refining the connection between intended learning outcomes and teaching and learning activities of Figure 1.

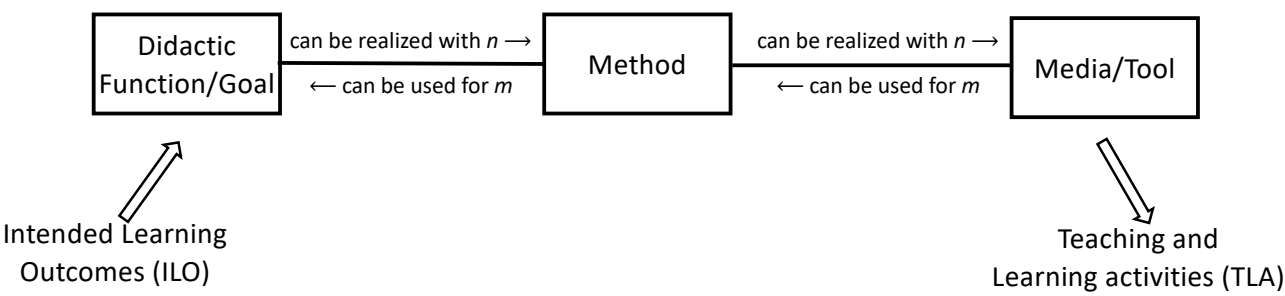

Figure 2. Architectural model refining the connection between ILO and TLA

We claim that the path from ILO to TLA must form an aligned chain from didactic goals via methods to media and tools. Note that didactic goals and teaching methods are interconnected by an m-to-n-relationship, indicating that for each goal there might be more than one appropriate method, and vice versa. The same holds true for the relation between methods and media or tools that can be used to put the methods to work. In the following, we focus on the different aspects in more detail.

\subsection{Didactic Goals}

Foremost, the selection of the sequence of didactic functions for a unit of teaching and learning is driven by the intended learning outcomes (ILOs) as described in (Biggs \& Tang, 2011). These ILOs can be considered as the roots of a design process leading to the teaching and learning activities. 
Analogous to ILOs that are formulated using verbs describing what the students are expected to perform, didactic goals are described using verbs describing the teacher's intention, i.e. "I want ..."

- to present factual knowledge to students

- to activate students (with respect to either content or in a mental, or in a physical manner)

- to design appropriate guiding questions for self/group learning

- to induce students to corporately work on a given problem

- to students to present a piece of work or information together

- to give feedback to students

- to receive feedback from students

- to assess students' knowledge (formative or summative)

- to induce students to reflect on something

- $\quad$ students to cooperatively solve a design task

Intentions like "I want to produce teaching videos" or "I want to use tool xyz" are no valid didactic goals since a selection of methods and adequate tools is part of a follow-up step.

\subsection{Teaching Methods}

Experience shows that many teachers tend to place teaching methods in the center of their course design, thus focusing on what they intend to be doing. However, in remote digital teaching scenarios, these teaching methods must be put into practice using appropriate digital environments and tools. This transition entails the risk that the whole design process will be driven by merely looking at the tools that appear to be quite cool, rather than focusing on didactic functions or goals.

Selecting specific methods and media which then will form a teaching and learning activity (unit) is driven by the physical setting in which the teaching and learning will take place. This physical setting must be considered with all its facets, not just restricted to the context of remote teaching scenarios imposed by the Covid pandemic. We differentiate two types of physical settings:

Asynchronous Setting, where students learn on their own, in their own pace and in any place, and

Synchronous Setting, where more than one person meets at the same time, and with or without a teacher being involved.

The synchronous setting can be further discriminated into three variants:

1. In-classroom teaching, i. e. the classical variant of teaching. 
2. Virtual teaching, where the class meets in a virtual classroom.

3. Hybrid variants, i. e. part of the class meets in a classroom and the lecture or other activities are simultaneously streamed or transmitted using a virtual classroom via a conferencing system.

Furthermore, teaching methods can be labeled or organized into clusters, such as gamification, engaging methods, or cooperative methods.

As a next step, a closer look at many modern teaching methods uncovers a kind of methodspecific intrinsic structure, as many methods indeed comprise a variety of sub-methods. We illustrate this on a small set of examples more or less randomly picked from the enormous amount of teaching methods that have been published up to now.

The methods Just-in-time teaching and Flipped Classroom (as many others) combine phases of self-regulated learning and interactive student-centered in-class activities (Bishop \& Verleger, 2013). Designing TLAs according to these methods requires a combination of several smaller methods, whose number is not even well designed by the method itself. The phase of self-regulated learning for example could require a presentation of factual or some kind of procedural knowledge. Subsequent in-class activities could require student activation, quizzes, or collaborative activities to apply the knowledge gained by self-regulated learning - and many more.

The Play Projects described by (Yakovleva \& Yakovlev, 2014) require several steps: i) selecting and fixing a research problem, ii) splitting students into (two) groups, iii) asking students to craft solutions, and finally iv) organizing a meeting where students then present and defend their solutions.

(Bescherer \& Spannagel, 2009) introduce - among several other patterns - the Technology on Demand Pattern. Implementation of this pattern requires the selection of an appropriate problem that has to meet given requirements, the design of appropriate questions, tasks and hints to guide students through a solution process requiring software tools.

\subsection{Media and Tools}

Finally, putting a method to work usually requires some kind of prepared media or material, as well as tools to either create these media or put them into usage. Again, we have an m-to$\mathrm{n}$ relationship between methods and media/tools, meaning that for each method usually more than one appropriate type of media and/or several tools can be considered, and most kinds of media and tools can be employed in more than one method.

In practice, sometimes only digital tools are considered and put to work, just because they are deemed to be cool. As well, it is important to clearly distinguish whether using a tool is 
in itself an explicit learning objective, or whether the tool is merely the means to achieve some other ILO.

\section{Examples}

As first example we elaborate on the didactic goal "I want to present factual knowledge to students". In terms of methods, this can be done by classical classroom teacher-centered lecturing; but also, by using videos or texts. Each of these variants requires a mapping to media and/or tools. Classroom teaching can make use of a blackboard or an electronic drawing facility. Videos and texts can be drawn from various sources, e. g. open educational resources, or can be produced by the teachers themselves. In the latter case a production process must be defined, which itself can require more or less complex tools and equipment depending on the degree of sophistication the teacher wishes to achieve. Note that these implementation steps are not explicitly visualized by Fig. 2, which merely provides a model overview.

For another example, the didactic goals "I want to students to present a piece of work or information together" and "I want students to cooperatively solve a design task" can be addressed with a method called whiteboarding as described in (Megowan-Romanowicz, 2016). When putting this method into practice, specific media and/or tools must be selected that are applicable in the intended setting. In principle, the example of whiteboarding opens both analogous and digital opportunities to the teacher. Here, analogous means that e.g. flipcharts can be used in classroom if that is the setting of choice. When considering digital settings, a whole bunch of tools opens up as summarized in (Bodnenko, Kuchakovska, Proshkin, \& Lytvyn, 2020). Having the palette of tools at hand, the pros and cons can be considered and a selection can be performed as described by Bodnenko et al. Then, the subsequent implementation step leads to either a classroom setting with prepared flipcharts or to a virtual setting with all prerequisites prepared.

\section{Conclusions and Further Work}

The presented architectural concept relates didactic intentions with methods and methods with tools, each in an n-to-m relationship. Designing teaching and learning activities according to this structure helps to focus on the respective didactic intention in a studentcentered manner. Incorporating assessments into the architecture is subject of future work. 


\section{References}

Bescherer, C., \& Spannagel, C. (2009). Design Patterns for the Use of Technology in Introductory Mathematics Tutorials. Education and Technology for a Better World (pp. 427--435). Berlin Heidelberg: Springer.

Biggs, J., \& Tang, C. (2011). Teaching for quality learning at university: What the student does. Pa.: MacGraw-Hill/Society for Research into Higher Education.

Bishop, J., \& Verleger, M. (2013). The flipped classroom: A survey of the research. ASEE Annual Conference and Exposition, Conference Proceedings.

Bodnenko, D. M., Kuchakovska, H. A., Proshkin, V. V., \& Lytvyn, O. S. (2020). Using a virtual digital board to organize student's cooperative learning. Proceedings of the 3rd International Workshop on Augmented Reality in Education (AREdu 2020), (pp. 357368). Kryvyi Rih, Ukraine.

Boling, E. C., Holan, E., Horbatt, B., Hough, M., Jean-Louis, J., Kurana, C., . . Spiezio, C. (2014). Using online tools for communication and collaboration: Understanding educators' experiences in an online course. The Internet and Higher Education, Vol. 23, pp. 48-55.

Brothers, P., \& Spies, M. (2020). Open-Source Taxonomy 2021. Retrieved from https:/www.globallearninglandscape.org/

Hamilton, E., Rosenberg, J., \& Akcaoglu, M. (2016, September). The Substitution Augmentation Modification Redefinition (SAMR) Model: a Critical Review and Suggestions for its Use. TechTrends, 60, pp. 433-441. doi:10.1007/s11528-016-0091-y

Lathan, J. (n.d.). The Complete List of Teaching Methods. (U. o. Diego, Editor) Retrieved 02 24, 2021, from https://onlinedegrees.sandiego.edu/complete-list-teaching-methods/

Megowan-Romanowicz, C. (2016). Whiteboarding: A Tool for Moving Classroom Discourse from Answer-Making to Sense-Making. The Physics Teacher, Vol. 54, pp. 8386.

Puentedura, R. (2006, August 18). Transformation, Technology, and Education. Retrieved 02 24, 2021, from http://hippasus.com/resources/tte/

Wissensmedien, L.-I. f. (Ed.). (n.d.). e-teaching.org.

Yakovleva, N., \& Yakovlev, E. (2014). Interactive teaching methods in contemporary higher education. Pacific Science Review, 16(2), 75-80. 